\title{
PEDIATRICS
}

\section{Clinical Spectrum, Morbidity, and Mortality in 113 Pediatric Patients With Mitochondrial Disease}

Fernando Scaglia, Jeffrey A. Towbin, William J. Craigen, John W. Belmont, E. O'Brian Smith, Stephen R. Neish, Stephanie M. Ware, Jill V. Hunter, Susan D. Fernbach, Georgirene D. Vladutiu, Lee-Jun C. Wong and Hannes Vogel Pediatrics 2004;114;925-931

DOI: $10.1542 /$ peds.2004-0718

\section{This information is current as of June 7, 2005}

The online version of this article, along with updated information and services, is located on the World Wide Web at: http://www.pediatrics.org/cgi/content/full/114/4/925

PEDIATRICS is the official journal of the American Academy of Pediatrics. A monthly publication, it has been published continuously since 1948. PEDIATRICS is owned, published, and trademarked by the American Academy of Pediatrics, 141 Northwest Point Boulevard, Elk Grove Village, Illinois, 60007. Copyright (C 2004 by the American Academy of Pediatrics. All rights reserved. Print ISSN: 0031-4005. Online ISSN: 1098-4275.

\section{American Academy of Pediatrics}




\title{
Clinical Spectrum, Morbidity, and Mortality in 113 Pediatric Patients With Mitochondrial Disease
}

\author{
Fernando Scaglia, $\mathrm{MD}^{*} \ddagger$; Jeffrey A. Towbin, MD $\ddagger \S$; William J. Craigen, $\mathrm{MD}, \mathrm{PhD}^{*} \ddagger$; \\ John W. Belmont, MD, PhD* ; E. O’Brian Smith, PhD\&; Stephen R. Neish, MD \$;;

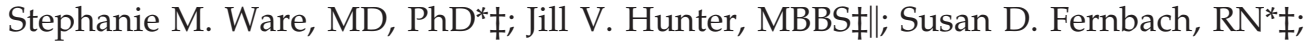 \\ Georgirene D. Vladutiu, PhDII; Lee-Jun C. Wong, PhD\#; and Hannes Vogel, MD**
}

\begin{abstract}
Objectives. The aim of this study was to elucidate the frequency of major clinical manifestations in children with mitochondrial disease and establish their clinical course, prognosis, and rates of survival depending on their clinical features.

Methods. We performed a retrospective review of the medical records of 400 patients who were referred for evaluation of mitochondrial disease. By use of the modified Walker criteria, only patients who were assigned a definite diagnosis were included in the study.

Results. A total of 113 pediatric patients with mitochondrial disease were identified. A total of $102(90 \%)$ patients underwent a muscle biopsy as part of the diagnostic workup. A significant respiratory chain (RC) defect, according to the diagnostic criteria, was found in $71 \%$ of the patients who were evaluated. In this cohort, complex I deficiency (32\%) and combined complex I, III, and IV deficiencies $(26 \%)$ were the most common causes of RC defects, followed by complex IV (19\%), complex III $(16 \%)$, and complex II deficiencies (7\%). Pathogenic mitochondrial DNA abnormalities were found in $11.5 \%$ of the patients. A substantial fraction $(40 \%)$ of patients with mitochondrial disorders exhibited cardiac disease, diagnosed by Doppler echocardiography; however, the majority $(60 \%)$ of patients had predominant neuromuscular manifestations. No correlation between the type of RC defect and the clinical presentation was observed. Overall, the mean age at presentation was 40 months. However, the mean age at presentation was 33 months in the cardiac group and 44 months in the noncardiac group. Twenty-six (58\%) patients in the cardiac group exhibited hypertrophic cardiomyopathy, 29\% had dilated cardiomyopathy, and the remainder (13\%) had left ventricular noncompaction. Patients with cardiomyopathy had an $18 \%$ survival rate at 16 years of age. Patients with neuromuscular features but no cardiomyopathy had a $95 \%$ survival at the same age.
\end{abstract}

From the *Department of Molecular and Human Genetics, Baylor College of Medicine, Houston, Texas; 掝as Children's Hospital, Houston, Texas; $\S$ Department of Pediatrics, Baylor College of Medicine, Houston, Texas; |Department of Radiology, Baylor College of Medicine, Houston, Texas; IDepartment of Pediatrics, School of Medicine \& Biomedical Sciences, State University of New York at Buffalo, Buffalo, New York, \#Institute for Molecular and Human Genetics, Georgetown University Medical Center, Washington, DC, **Department of Pathology, Stanford University, Stanford, California

Accepted for publication Jun 14, 2004.

doi:10.1542/peds.2004-0718

Reprint requests to (F.S.) Department of Molecular and Human Genetics, Baylor College of Medicine, One Baylor Plaza, Houston, TX 77030. E-mail fscaglia@bcm.tmc.edu

PEDIATRICS (ISSN 0031 4005). Copyright (C) 2004 by the American Academy of Pediatrics.
Conclusions. This study gives strong support to the view that in patients with RC defects, cardiomyopathy is more common than previously thought and tends to follow a different and more severe clinical course. Although with a greater frequency than previously reported, mitochondrial DNA mutations were found in a minority of patients, emphasizing that most mitochondrial disorders of childhood follow a Mendelian pattern of inheritance. Pediatrics 2004;114:925-931; mitochondrial encephalomyopathies, mitochondrial cardiomyopathies, modified Walker criteria, morbidity, survival rate.

ABBREVIATIONS. RC, respiratory chain; mtDNA, mitochondrial DNA; SDH, succinate dehydrogenase; MELAS, mitochondrial encephalopathy with lactic acidosis and stroke-like episodes; MERRF, myoclonic epilepsy with ragged red fibers; NARP, neuropathy, ataxia, and retinitis pigmentosa; LHON, Leber hereditary optic neuropathy; MNGIE, mitochondrial neurogastrointestinal encephalomyopathy; LVNC, left ventricular noncompaction; HCM, hypertrophic cardiomyopathy; NADH-TR, nicotinamide adenine dinucleotide-tetrazolium reductase.

$\mathrm{T}$ The first mitochondrial disorder was recognized in $1962^{1}$; since then, mitochondrial disorders have emerged as major clinical entities. Mitochondrial encephalomyopathies are a genetically, biochemically, and clinically heterogeneous group of disorders associated with abnormalities of oxidative phosphorylation. Organs such as the brain, heart, and skeletal muscle are highly energy dependent and vulnerable to defects in energy metabolism. Mitochondrial respiratory chain (RC) disorders are believed to occur with an incidence of 1/10 000 live births. ${ }^{2,3}$ The minimum birth prevalence for onset at any age of an RC defect has been estimated at 1/7634. ${ }^{4}$ Pathogenic mitochondrial DNA (mtDNA) mutations are found in many adults with a suspected RC defect. ${ }^{5}$ However, mtDNA mutations are identified in only a small proportion of pediatric patients, and the diagnosis of pediatric mitochondrial disorders relies mostly on enzymatic investigations, with an often-difficult interpretation. ${ }^{6}$ Hence, establishing a reliable diagnosis is more difficult for pediatric RC defects than those with onset in adulthood. ${ }^{7}$ Therefore, diagnostic criteria for pediatric mitochondrial disorders have been modified from an adult classification system, ie, the modified Walker criteria. ${ }^{8}$ Because the natural history of this heterogeneous group of disorders remains largely unknown, the aim of the present study was to assess the frequency of major 
clinical manifestations at presentation in a population of infants and children with definite diagnosis of mitochondrial disease on the basis of the modified Walker criteria. We sought to understand their clinical course and outcome depending on their different presenting clinical features. We have identified distinct clinical courses and outcomes for pediatric patients with mitochondrial disease presenting with cardiac versus noncardiac findings. These results will allow for improved management and counseling of patients with mitochondrial disease.

\section{METHODS}

A retrospective review of the genetic, hospital, and laboratory records of 400 children who had neurologic and musculoskeletal features and were referred to Texas Children's Hospital between 1997 and 2003 for investigation of mitochondrial disease was conducted. The diagnosis was established according to an objective classification scheme on the basis of assigning major or minor criteria for clinical, pathologic, enzymatic, molecular, and metabolic parameters (Table 1). Patients with 2 major or 1 major and 2 minor criteria were assigned with a definite diagnosis and were included in the study. Functional assays (fibroblast adenosine triphosphate synthesis and ability of fibroblasts to grow in galactose media) are available only on a research basis and were not routinely used in our study. A muscle biopsy was performed as part of the diagnostic workup in the majority of patients when a known pathogenic mtDNA mutation was not identified in blood. The procedure was conducted in the operating room, and the tissue was snap-frozen in isopentane cooled to $-100^{\circ} \mathrm{C}$ for $\sim 30$ minutes before the biopsy. A standard diagnostic panel using cryosections was performed in each case, including hematoxylin and eosin, modified Gomori trichrome, lipid stains, succinate dehydrogenase (SDH), and cytochrome oxidase enzyme histochemistry. Electron microscopy was performed in only 78 (69\%) tissue samples. Mitochondrial enzyme assays were performed in $5 \%$ homogenates of frozen muscle specimens using standard spectrophotometric assays adapted from previous methods used to evaluate the activity of RC complexes in animal tissues. ${ }^{9-12} \mathrm{RC}$ assays were not conducted in fibroblasts. All patients were investigated for mtDNA deletions/duplications, and common point mutations (mitochondrial encephalopathy with lactic acidosis and stroke-like episodes [MELAS] A3243G and T3271C; myoclonic epilepsy with ragged red fibers [MERRF] A8344G and T8356C; neuropathy, ataxia and retinitis pigmentosa [NARP] T8993G and T8993C; cardiomyopathy G8363A; and Leber hereditary optic neuropathy [LHON] G11778A, G3460A, T14484C, and G14459A). ${ }^{13,14}$ Patients with pathogenic mtDNA mutations (MELAS, MERRF, LHON, NARP, and chronic progressive ophthalmoplegia) were summarized into a group named classical mitochondrial syndromes. Mitochondrial neurogastrointestinal encephalomyopathy [MNGIE] was characterized by severe gastrointestinal dysmotility presenting as repeated attacks of vomiting and pseudo-obstruction, cachexia following dysmotility, ptosis, external ophthalmoparesis, peripheral neuropathy, and leukoencephalopathy and was finally confirmed by mutations in the thymidine phosphorylase gene. Lethal infantile mitochondrial disease was characterized by a neonatal onset and a fulminant course (death within 6 months of life). The diagnosis of Leigh syndrome was established by neuropathologic and clinical findings. Cardiomyopathy and myopathy was a clinical subtype dominated by cardiac and myopathic symptoms. Within this group, Sengers syndrome was diagnosed with the presence of congenital cataracts, hypertrophic cardiomyopathy, mitochondrial myopathy, and lactic acidosis; and Barth syndrome was diagnosed with the presence of left ventricular noncompaction (LVNC), skeletal myopathy, 3-methylglutaconic aciduria, and diminished statural growth. The group of nonspecific encephalomyopathy included all patients who would not fit into any of the other subtypes. The clinical parameters that were analyzed included developmental delay, abnormalities of tone, movement disorder, seizures, cardiomyopathy, arrhythmia, ophthalmologic disease, and hearing loss. The diagnostic parameters that were analyzed included lactic acidosis (documented with $>1$ sample the same day), central nervous system imaging abnormalities, histopathologic evidence of mitochondrial proliferation (see Discussion section suggesting mitochondrial proliferation), cytochrome c oxidase deficiency by enzyme histochemistry, ultrastructural abnormalities of mitochondria, and type of $\mathrm{RC}$ defect by quantitative biochemical analysis.

For evaluating outcome, patients were divided in 2 groups: 1 with cardiomyopathy and myopathy as main clinical presentation and 1 with neuromuscular features but without cardiac involvement. Kaplan Meier survival analyses were performed using SPSS (SPSS Inc, Chicago, IL) to study survival rates in the cardiac and noncardiac groups. The rates were compared using a log-rank test. A $t$ test for independent groups was used to compare means and a $\chi^{2}$ test was used to compare proportions between cardiac and noncardiac groups.

\section{RESULTS}

We identified 113 patients with definite mitochondrial disease by use of the modified Walker criteria

TABLE 1. Modified Walker Criteria Applied to Children Who Were Referred for Evaluation of Mitochondrial Disease $(N=400)^{*}$

\begin{tabular}{|c|c|c|}
\hline & Major Criteria & Minor Criteria \\
\hline Clinical & $\begin{array}{l}\text { Clinically complete RC encephalomyopathyt or } \\
\text { a mitochondrial cytopathy defined as fulfilling } \\
\text { all } 3 \text { of the following } \ddagger\end{array}$ & Symptoms compatible with a RC defect§ \\
\hline Histology & $>2 \%$ ragged red fibers in skeletal muscle & $\begin{array}{l}\text { Smaller numbers of RRF, SSAM, or widespread electron } \\
\text { microscopy abnormalities of mitochondria }\end{array}$ \\
\hline Enzymology & $\begin{array}{l}\text { Cytochrome c oxidase negative fibers or residual } \\
\text { activity of a RC complex }<20 \% \text { in a tissue; } \\
<30 \% \text { in a cell line, or }<30 \% \text { in } 2 \text { or more } \\
\text { tissues }\end{array}$ & $\begin{array}{l}\text { Antibody-based demonstration of an RC defect or } \\
\text { residual activity of an RC complex } 20 \%-30 \% \text { in a } \\
\text { tissue, } 30 \%-40 \% \text { in a cell line, or } 30 \%-40 \% \text { in } 2 \text { or } \\
\text { more tissues }\end{array}$ \\
\hline Functional & $\begin{array}{l}\text { Fibroblast ATP synthesis rates }>3 \text { SD below } \\
\text { mean }\end{array}$ & $\begin{array}{l}\text { Fibroblast ATP synthesis rates } 2-3 \text { SD below mean, or } \\
\text { fibroblasts unable to grow in galactose media }\end{array}$ \\
\hline Molecular & $\begin{array}{l}\text { Nuclear or mtDNA mutation of undisputed } \\
\text { pathogenicity }\end{array}$ & Nuclear or mtDNA mutation of probable pathogenicity \\
\hline Metabolic & & $\begin{array}{l}\text { One or more metabolic indicators of impaired metabolic } \\
\text { function }\end{array}$ \\
\hline
\end{tabular}

ATP indicates adenosine triphosphate; SSAM, subsarcolemmal accumulation of mitochondria.

* This classification scheme was applied to 400 patients who were referred for evaluation of mitochondrial disease. The details of these criteria are described by Bernier et al. ${ }^{8}$

+ Leigh disease, Alpers disease, LIMD, Pearson syndrome, Kearns-Sayre syndrome, MELAS, MERRF, NARP, MNGIE, and LHON. $\ddagger 1$ ) Unexplained combination of multisystemic symptoms that is essentially pathognomic for an RC disorder, 2) a progressive clinical course with episodes of exacerbation or a family history strongly indicative of a mtDNA mutation, 3) other possible metabolic or nonmetabolic disorders have been excluded by appropriate testing.

$\S$ Added pediatric features: stillbirth associated with a paucity of intrauterine movement, neonatal death or collapse, movement disorder, severe failure to thrive, neonatal hypotonia, and neonatal hypertonia as minor clinical criteria. 
(58\% male and $42 \%$ female), with ages ranging between 2 weeks and 18 years. The gender ratio in our entire series (1.4) exhibited a slight male predominance $(P=.09)$. The mean age at presentation was 40 months, and the mean age at death was 5 years and 4 months. Most patients were of white $(46 \%)$ and Hispanic (33\%) ancestry. The rest of the ethnic distribution was a follows: black, 9\%; Southeast Asian, $6 \%$; Native American, 2\%; and a group of patients from the Arabian Peninsula, 4\%. Mitochondrial inheritance was observed in 13 patients. Autosomal recessive inheritance could be established by the finding of nuclear DNA mutations in 2 patients. $X-$ linked inheritance was established in 1 case of Barth syndrome. Ninety-seven cases were considered sporadic. A total of $102(90 \%)$ patients underwent a muscle biopsy as part of the diagnostic workup. Eleven patients did not undergo a muscle biopsy because they were found to have a classical mitochondrial syndrome and a pathogenic mtDNA mutation was found in blood, fulfilling clinical and molecular criteria for definite mitochondrial disease (1 patient with NARP, 1 patient with LHON, 1 patient with MERRF, and 8 patients with MELAS). The patients were classified by clinical criteria into 6 phenotypes (Table 2).

\section{Biochemical Defect}

A significant RC defect was found in $71 \%$ of the patients. In this cohort, we found that complex I deficiency (32\%) and combined complex I, III, and IV deficiencies $(26 \%)$ were the most common causes of RC defects, followed by complexes IV (19\%), III $(16 \%)$, and II deficiencies (7\%; Fig 1).

\section{Molecular Defect}

Pathogenic mtDNA and nuclear DNA mutations were found in $13(11.5 \%)$ and $3(2.6 \%)$ of the patients, respectively. The most common mtDNA mutation in our group was the $3243 \mathrm{~A}>\mathrm{G}$ MELAS mutation observed in 8 patients, followed by the $8344 \mathrm{~A}>\mathrm{G}$ MERRF mutation observed in 2 patients (Table 3). The nuclear DNA mutations were a homozygous splice site mutation (IVS1-1G $>$ C) in the thymidine phosphorylase gene in 1 patient with MNGIE, a missense mutation (G197R) in the G4.5 gene in 1 patient with Barth syndrome, and 2 compound heterozygous mutations (Q195X/A56G) in the SURF-1 gene in 1 patient with Leigh syndrome and cytochrome $c$ oxidase deficiency. There was a statistically significant difference in the mean age at presentation in the group with identified pathogenic mtDNA mutations

TABLE 2. Clinical Subtypes in the Cohort

\begin{tabular}{lc}
\hline \multicolumn{1}{c}{ Clinical Subtypes } & $n(N=113)$ \\
\hline Nonspecific encephalomyopathy & 44 \\
Leigh syndrome & 6 \\
Lethal infantile mitochondrial disease & 4 \\
Classical mitochondrial syndromes & 13 \\
Mitochondrial neurogastrointestinal & 1 \\
encephalomyopathy & 45 \\
Cardiomyopathy and myopathy & \\
\hline
\end{tabular}

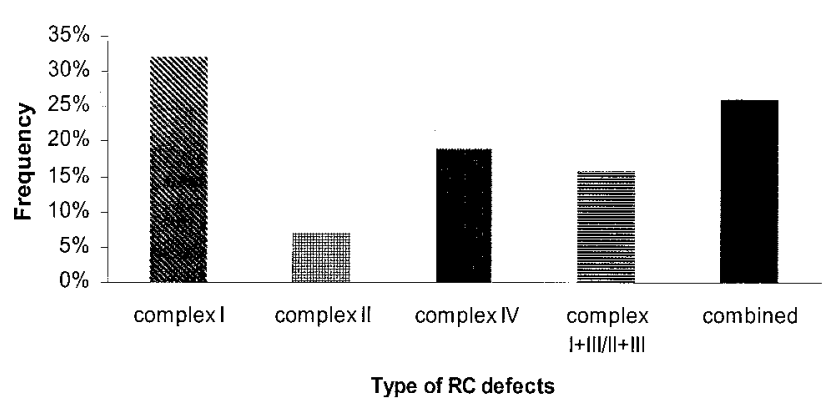

Fig 1. Comparative frequencies of RC defects.

TABLE 3. Classical Mitochondrial Syndromes and mtDNA Mutations of Undisputed Pathogenicity

\begin{tabular}{ccc}
\hline $\begin{array}{c}\text { Subtypes of Classical } \\
\text { Mitochondrial Syndromes }\end{array}$ & $\begin{array}{c}\text { MtDNA } \\
\text { Mutation }\end{array}$ & $n(N=13)$ \\
\hline MELAS & $3243 \mathrm{~A}>\mathrm{G}$ & 8 \\
MERRF & $8344 \mathrm{~A}>\mathrm{G}$ & 2 \\
LHON & $3460 \mathrm{G}>\mathrm{A}$ & 1 \\
NARP & $8993 \mathrm{C}>\mathrm{T}$ & 1 \\
CPEO & $5-\mathrm{kb}$ deletion & 1 \\
\hline
\end{tabular}

versus the rest of the cohort (110 months vs 32 months, respectively; $P<.0001)$.

\section{Clinical Presentation}

The predominant clinical presentations were cardiomyopathy and myopathy in 45 patients and nonspecific encephalopathy in 44 patients. The cardiomyopathy group included 1 patient with Sengers syndrome and 1 patient with Barth syndrome. There was a significantly different mean age at presentation in the cardiac group versus the noncardiac group (33 vs 44 months, respectively; $P<.005$ ).

No statistically significant associations between the type of RC defect and the clinical presentation (cardiac vs noncardiac) or the different clinical parameters studied were noted. A substantial fraction $(40 \%)$ of patients with RC defects exhibited cardiac disease, diagnosed by Doppler echocardiography; however, the majority $(60 \%)$ of patients had predominant noncardiac manifestations. Moderate to severe developmental delay was observed at presentation in $100 \%$ of the patients with noncardiac phenotype. In the group with noncardiac phenotype, different clinical parameters were studied at presentation. Twenty-two (32\%) patients had ophthalmologic manifestations of mitochondrial disease. Sensorineural hearing loss was detected in $21 \%$ of the patients, and it was not associated with the mitochondrial A1555G 12S ribosomal RNA mutation. Fifty-four $(79 \%)$ patients exhibited hypotonia, $20 \%$ hypertonia, $50 \%$ seizures, $12 \%$ movement disorders, $6 \%$ ataxia, and $70 \%$ abnormal brain magnetic resonance imaging findings.

In the group with cardiac manifestations, $58 \%$ of the patients exhibited hypertrophic cardiomyopathy (HCM), 29\% dilated cardiomyopathy, and 13\% LVNC. Only $11 \%$ of the patients exhibited cardiac arrhythmias, with ventricular tachycardia the most commonly observed arrhythmia. Wolf-Parkinson- 
White syndrome was observed in only 2 patients. Mild cognitive deficits including decreased performance IQ and short-term memory deficits were observed in $20 \%$ of the patients in the cardiomyopathy and myopathy group.

Sixty-eight $(60 \%)$ patients with definite mitochondrial disease exhibited an elevation of plasma lactic acid level sampled on $>1$ occasion. Mitochondrial ultrastructural abnormalities were noted in $83 \%$ of the cases when electron microscopy was performed. Only $56 \%$ of the patients who underwent muscle biopsy had evidence of mitochondrial proliferation or increased subsarcolemmal staining when the reduced nicotinamide adenine dinucleotide-tetrazolium reductase (NADH-TR) and $\mathrm{SDH}$ stains were evaluated; $7 \%$ of them exhibited ragged red fibers, and by enzyme histochemistry, focal absence of cytochrome c oxidase activity was found in $13 \%$ of the patients.

Survival was measured in the groups with and without cardiomyopathy at the age of 16 years, and the differences between the 2 groups are strikingly illustrated in the Kaplan-Meier survival analysis (Fig 2). Patients with cardiac manifestations had an $18 \%$ survival. This outcome was significantly different $(P$ $<$.0001) from the outcome of patients with noncardiac features, who had a $95 \%$ survival at the same age.

\section{DISCUSSION}

By using the modified Walker criteria, we were able to gather a large group of pediatric patients with a definite diagnosis of a mitochondrial disorder and study their clinical histories. Overall, the frequency of RC defects in our cohort of pediatric patients with mitochondrial disorders was similar, albeit slightly lower, than that previously reported. ${ }^{15}$ Among the patients with mitochondrial disorders and normal skeletal muscle RC enzyme activities, we observed 1

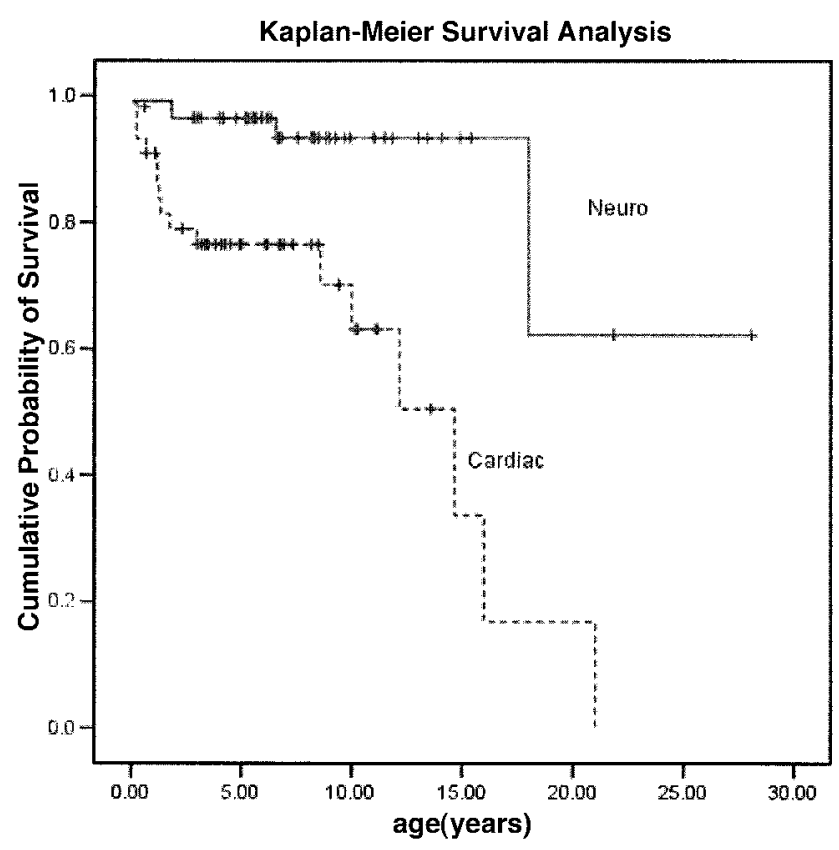

Fig 2. Kaplan Meier survival analysis. patient with MERRF and 1 patient with MNGIE with striking neuromuscular phenotypes. ${ }^{16}$ The finding of normal RC activity in skeletal muscle, in the presence of clinical neuromuscular manifestations, does not rule out mitochondrial disease, and a thorough diagnostic workup should be pursued, because compensatory amplification of mitochondrial DNA resulting in normal RC activity has been observed in mitochondrial disorders. ${ }^{17}$ As we have observed in the case of the patient with MNGIE, who exhibited normal mitochondrial enzymes, and muscle morphology, 16 the final diagnosis relied on clinical and molecular criteria. We think that there is no absolute gold standard to establish a diagnosis of mitochondrial encephalomyopathy, and enzymology is not necessarily more sensitive than histology. All diagnostic criteria equally contribute to solve the diagnostic puzzle.

In our cohort, complex I deficiency was the most commonly observed RC defect, a finding similar to that of previous studies. ${ }^{18,19}$ The second most common was the combined deficiencies of complex I, III, and IV. The incidence of complex IV deficiency in our clinic population was much lower than that observed in a previous study. ${ }^{18}$ However, the different ethnic composition and the remarkable high rate of parental consanguinity observed by von Kleist-Retzow et $\mathrm{al}^{18}$ could account for the higher incidence of complex IV deficiency in their patient sample. Overall, our results are also different from those reported by Rubio-Gozalvo et al, ${ }^{20}$ who observed combined $\mathrm{RC}$ defects as the most frequent RC defect. Because the activity of complex III was not measured directly, the combined deficiencies of complex I+III and II + III in our cohort could also reflect muscle coenzyme $\mathrm{Q}_{10}$ deficiency in addition to isolated complex III deficiency. ${ }^{21}$ The observed gender ratio, without reaching statistical significance, suggested the possibility of X-linked inheritance in some of our families. One complex I subunit gene, the NDUFA1 gene, has been mapped to the $X$ chromosome. ${ }^{22}$ In contrast to previous studies, ${ }^{18,19}$ the gender ratio in our group with complex I deficiency was not skewed toward boys.

mtDNA point mutations and deletions/duplications were found in a minority $(11.5 \%)$ of patients in our cohort, suggesting that most cases of pediatric mitochondrial disorders are caused by mutations in nuclear genes. This result is 2 -fold higher than that reported in a previous study of 2000 patients with mitochondrial disorders, in which using a similar method, only $5.4 \%$ of the patients carried identifiable, disease-causing mutations. ${ }^{13}$ This difference could be explained by more stringent selection criteria in our study. The percentage of patients with pathogenic mtDNA mutations could be significantly higher in our sample, because we tested only for known mtDNA mutations rather than screening the entire mitochondrial genome. It should be noted that mutation analysis of the entire mitochondrial genome has identified novel mtDNA mutations in $\sim 10 \%$ of the patients evaluated in another study. ${ }^{23}$ Patients with mitochondrial disorders as a result of a pathogenic mtDNA mutation became symptomatic 
at an older age when compared with the rest of the group, supporting the hypothesis that there are clinical differences in patients with mitochondrial cytopathies as a result of nuclear versus mtDNA mutations. ${ }^{20}$

Our observation that not all patients with definite mitochondrial disorders exhibit elevated plasma lactic acid levels is consistent with previous studies. ${ }^{19,24}$ This commonly used screening test, therefore, is not a sensitive marker of mitochondrial dysfunction. Lactic acidosis is one of the criteria often used to initiate additional investigation of $\mathrm{RC}$ defects, particularly skeletal muscle enzymology. It has been recommended to measure plasma lactate levels on $>1$ occasion throughout the day in fasting and nonfasting conditions 55,26 ; however, lactic acidosis should not be considered a prerequisite for diagnosis because serum lactic acid is not a reliable marker for mitochondrial dysfunction. There are mitochondrial diseases, such as NARP and its infantile variant maternally inherited Leigh syndrome, in which blood lactate is often normal or mildly increased at best. ${ }^{27}$ In conclusion, the absence of lactic acidosis should not preclude any additional diagnostic workup for $\mathrm{RC}$ defects.

Although ultrastructural mitochondrial abnormalities were found in the majority of patients with mitochondrial disease, skeletal muscle samples from $17 \%$ of the patients had normal ultrastructural findings. Osmiophilic or paracrystalline inclusions and distinctly abnormal (circular, disoriented, rarified) cristae in the context of markedly increased mitochondrial size and abnormal shape could be considered diagnostic ultrastructural findings of mitochondrial disease. However, morphologic changes in mitochondria (isolated mitochondrial enlargement or proliferation) are not necessarily specific and are not restricted to syndromes that result from primary errors in mitochondrial function. ${ }^{19,28,29}$ In addition, some patients with nonspecific ultrastructural mitochondrial abnormalities (isolated mitochondrial enlargement or proliferation) did not meet criteria for inclusion in our study because they did not exhibit other diagnostic criteria for mitochondrial disease.

Other histologic findings suggestive of mitochondrial proliferation, eg, increased subsarcolemmal staining by NADH-TR and SDH, were lacking in $35 \%$ of the patients with proven mitochondrial dysfunction. This lack of evidence of proliferation correlated with a normal activity of citrate synthase, a mitochondrial matrix enzyme frequently used as an index of mitochondrial proliferation. The NADH-TR stain detects a reductase that is present in both mitochondria and sarcoplasmic reticulum and therefore is not a reliable marker for mitochondrial proliferation. This stain is not meant to assess complex I (NADH dehydrogenase) activity. The SDH stain can be used as a more sensitive detector of subtle mitochondrial proliferation in the form of blue rims at the periphery of muscle fibers. This stain is not affected by mtDNA mutations; therefore, it is an excellent marker of mitochondrial mass. ${ }^{30}$ However, as demonstrated in our study, even with proven mitochondrial dysfunction by enzymology and clinical crite- ria, the SDH stain might not reveal proliferation. Ragged red fibers were noted in a minority of patients. In our experience, it is very unusual to find ragged red fibers in children younger than 3 years. ${ }^{29}$ The observed focal absence of cytochrome c oxidase deficiency could suggest heteroplasmy in patients who harbor mtDNA mutations. It was noted in 1 patient with chronic progressive external ophthalmoplegia caused by a mtDNA deletion and in 1 patient with MERRF caused by the A8344G mtDNA mutation. Some other patients who exhibited focal absence of cytochrome c oxidase were not found to have pathogenic mtDNA mutations by the screen performed in this study; however, these patients could have other pathogenic mtDNA mutations because complete sequencing of the mtDNA genome was not conducted. Overall, it is necessary to consider that normal muscle morphology does not rule out the diagnosis of mitochondrial dysfunction because some patients with defined errors in mitochondrial metabolism may lack mitochondrial ultrastructural abnormalities and, conversely, that mitochondrial proliferation or even cytochrome c oxidase deficiency by enzyme histochemistry may be seen in non-RC defects. ${ }^{29}$

Of the clinical parameters studied in our cohort, none showed a statistically significant correlation with a particular RC defect. This observation was also made in a previous study. ${ }^{18}$ Classical mitochondrial syndromes in our group were rare. They have been found to occur more commonly outside childhood. ${ }^{31,32}$ Nonspecific encephalomyopathy was a frequent finding in our group, as reported elsewhere. $^{20}$

Cardiac involvement has been reported to be frequent in mitochondrial disorders. $3,33-35$ Little is known about the natural history of mitochondrial cardiomyopathy in children. Previous studies have indicated a frequency of cardiomyopathy of $18 \%$ to $25 \%$ in children with mitochondrial encephalomyopathies. ${ }^{3,35}$ In our study, a substantial fraction $(40 \%)$ of the patients with RC defects exhibited cardiomyopathy, giving strong support to the notion that it is more common than previously believed in these disorders.

In 1 recent study, all patients with mitochondrial cardiomyopathies exhibited HCM. ${ }^{35}$ However, earlier reports had documented both dilated and HCM occurring in mitochondrial cytopathies, with the latter more frequently associated with mitochondrial disease, ${ }^{33}$ consistent with our observations. Previous reports suggest that in some cases, dilation is simply a later stage in the process, although prospective longitudinal studies are needed. ${ }^{36,37}$

LVNC was also found in our cohort, expanding the spectrum of mitochondrial cardiomyopathies. LVNC has been recognized as a distinct form of cardiomyopathy and is thought to represent an arrest in endomyocardial morphogenesis, characterized by the presence of numerous prominent trabeculations and deep intertrabecular recesses within the left ventricle. ${ }^{38}$ Isolated LVNC has been found to be allelic with Barth syndrome, ${ }^{39}$ an X-linked recessive disorder associated with dilated cardiomyopa- 
thy, skeletal myopathy, neutropenia, and short stature in male individuals. ${ }^{40}$ In patients with Barth syndrome, mutations in the G4.5 gene have been identified. ${ }^{41}$ Only 2 patients of our cohort who presented with LVNC were male, and a G4.5 mutation has been identified in 1 of them.

The true incidence of cognitive deficits in mitochondrial disorders remains unknown. Developmental delay was present in a substantial percentage $(68 \%)$ of our patients. Even a fraction $(20 \%)$ of patients who presented with a cardiomyopathy exhibited mild cognitive delays, including compromised visuospatial abilities and short-term memory deficits. This observation confirms a previous study ${ }^{42}$ revealing that $\sim 75 \%$ of the patients with mitochondrial encephalomyopathies had higher cerebral dysfunction and intellectual deterioration. A second study also found a higher incidence of cognitive deficits $(60 \%)$ in a group of children with mitochondrial disorders. ${ }^{43}$ Performance IQ is lower than verbal IQ in these disorders, with demonstrated nonverbal cognitive impairment and compromised visuospatial abilities. ${ }^{44}$ Because of the frequency of developmental delay observed in our study and previous reports, we think that it is relevant to pursue mitochondrial studies in patients with developmental delay, seizures, and hypotonia, which do not clinically fall within the known mitochondrial encephalomyopathies. This conclusion is further supported by the results of other investigators, who have suggested that $15 \%$ of patients with unexplained psychomotor retardation have a probable mitochondrial disease. ${ }^{45}$

Patients with RC defects exhibit movement disorders, in particular dystonia. A specific missense mutation in the mtDNA complex I (NADH dehydrogenase) gene, MTDN6, has been linked to maternally inherited dystonia accompanied by LHON. ${ }^{46}$ Defects in complex I have been associated with other cases of dystonia, and a survey of mitochondrial OXPHOS complexes in platelet mitochondria revealed that patients with focal dystonia had an average of a $37 \%$ reduction in complex I activity. ${ }^{47}$ Dystonia was the most frequently observed movement disorder in our patients. However, in our group, there was no particular association between dystonia and complex I deficiency.

The spectrum of ophthalmologic manifestations of mitochondrial disease in our study included optic atrophy, ptosis, nystagmus, pigmentary retinopathy, and ophthalmoplegia. The percentage observed in our study (53\%) is lower than that previously reported. ${ }^{15}$ That not all patients received an ophthalmologic evaluation could explain in part the lower frequency of ophthalmologic manifestations observed in our group.

The frequency of patients who had hearing loss is similar to a previous report in which hearing loss was documented in $19.8 \%$ of patients with mitochondrial disease. ${ }^{48}$ Nevertheless, it was substantially lower than that found in another study, in which $80 \%$ of the patients who underwent testing had hearing loss or significant auditory dysfunction, making it the most common clinical finding associated with mitochondrial disease. ${ }^{49}$ Our finding could represent an underestimation, because not all of the patients included in our study underwent formal hearing testing. However, different diagnostic and age criteria were applied to evaluate patients with mitochondrial disease in these previous studies. ${ }^{49}$

Brain imaging studies in our group showed a wide spectrum of abnormalities that included cerebellar and cortical atrophy, brainstem and basal ganglia lesions, agenesis of the corpus callosum, and white matter abnormalities. Isolated posterior fossa abnormalities with cerebellar volume loss were found in $25 \%$ of the patients with abnormal neuroimaging studies. Previous reports have suggested that deficiency of cytochrome c oxidase deficiency and other $\mathrm{RC}$ disorders have to be considered in the differential diagnosis of cerebellar hypoplasia. ${ }^{50}$

By using the modified Walker criteria, we were able to study the clinical history of a large group of pediatric patients with a definite diagnosis of a mitochondrial disorder. We conclude that in our cohort of patients, complex I deficiency and combined RC deficiency are the most commonly observed RC defects. mtDNA mutations and deletions are found in a minority of pediatric patients, emphasizing that most pediatric cases probably have a Mendelian recessive inheritance. Classical mitochondrial syndromes are not frequently found in pediatric mitochondrial disorders. They tend to present at an older age when compared with the rest of the group. The results of the present study strongly suggest that the frequency of cardiomyopathy in pediatric mitochondrial disorders is higher than previously believed. In addition, cardiac function in mitochondrial patients deteriorates rapidly regardless of the associated $\mathrm{RC}$ defect. Early and aggressive supportive treatment might increase the chances of survival.

Our study demonstrates that patients with a noncardiac presentation generally survive longer than patients who present with cardiomyopathy. Overall, the frequency of mental retardation in patients with mitochondrial disorders is high, suggesting a pivotal role for mitochondrial integrity in cognitive function.

Even in the presence of normal mitochondrial enzymes, histopathologic evidence of mitochondrial disease, in addition to a classical clinical presentation, could suggest a definite mitochondrial disease; and the absence of histopathologic evidence, in the context of RC enzyme deficiency, would not rule out these conditions. Pediatric mitochondrial disorders can be accompanied by normal muscle morphology, normal plasma lactate, normal mitochondrial enzymes on skeletal muscle, normal mtDNA mutation screening, and a nonclassical clinical presentation because none of these criteria has absolute sensitivity to detect mitochondrial disease. Faced with the absence of perfect diagnostic criteria for the diagnosis of mitochondrial disease, the modified Walker criteria constitute the best diagnostic tools currently available. When the index of suspicion is high, an integrated approach is required for the diagnosis of mitochondrial cytopathies.

\section{ACKNOWLEDGMENT}

We recognize the support of the Mental Retardation Research Center of Baylor College of Medicine. 


\section{REFERENCES}

1. Luft R, Ikkos D, Palmieri G, Ernster L, Afzelius B. A case of severe hypermetabolism of non-thyroid origin with a defect in the maintenance of mitochondrial respiratory control. A correlated clinical, biochemical and morphological study. J Clin Invest. 1962;41:1776-1804

2. Applegarth DA, Toone JR, Lowry RB. Incidence of inborn errors of metabolism in British Columbia, 1969-1996. Pediatrics. 2000;105(1). Available at: www.pediatrics.org/cgi/content/full/105/1/e10

3. Darin N, Oldfors A, Moslemi AR, Holme E, Tulinius M. The incidence of mitochondrial encephalomyopathies in childhood: clinical features and morphological, biochemical and DNA abnormalities. Ann Neurol. 2001;49:377-383

4. Skladal D, Halliday J, Thornburn DR. Minimum birth prevalence of mitochondrial respiratory chain disorders in children. Brain. 2003;126: 1905-1912

5. Chinnery PF, Turnbull DM. Mitochondrial medicine. QJM. 1997;90: 657-667

6. Thornburn DR, Smeitink J. Diagnosis of mitochondrial disorders: clinical and biochemical approach. J Inherit Metab Dis. 2001;24:312-316

7. Thornburn DR. Practical problems in detecting abnormal mitochondrial function and genomes. Hum Reprod. 2000;15:57-67

8. Bernier FP, Boneh A, Dennett X, Chow CW, Cleary MA, Thornburn DA. Diagnostic criteria for respiratory chain disorders in adults and children. Neurology. 2002;59:1406-1411

9. King TE, Howard RL. Preparation and properties of NADH dehydrogenase from cardiac muscle. In: Estabrook R, Pullman M, eds. Methods in Enzymology: Oxidation and Phosphorylation. New York, NY: Academic Press; 1967:275-294

10. King TE. Preparation of succinate dehydrogenase and reconstitution of succinate oxidase. In: Estabrook R, Pullman M, eds. Methods in Enzymology: Oxidation and Phosphorylation. New York, NY: Academic Press; 1967:322-331

11. Yonetan T. Cytochrome oxidase: beef heart. In: Estabrook R, Pullman M, eds. Methods in Enzymology: Oxidation and Phosphorylation. New York, NY: Academic Press; 1967:332-335

12. Srere PA. Citrate synthase. In: Lowenstein J, ed. Methods in Enzymology: Oxidation and Phosphorylation. New York, NY: Academic Press; 1969: 3-11

13. Liang $\mathrm{MH}$, Wong L-JC. Yield of mtDNA mutation analysis in 2000 patients. Am J Med Genet. 1998;77:385-400

14. Wong L-JC, Senadheera D. Direct detection of multiple point mutations in mitochondrial DNA. Clin Chem. 1997;43:1857-1861

15. Skladal D, Sudmeier C, Konstantopoulou V, et al. The clinical spectrum of mitochondrial disease in 75 pediatric patients. Clin Pediatr. 2003;42: 703-710

16. Szigeti K, Wong LJ-C, Perng CL, et al. MNGIE with lack of skeletal muscle involvement and a novel TP splice site mutation. J Med Genet. 2004;41:125-129

17. Wong L-JC, Perng CL, Hsu CH, et al. Compensatory amplification of mitochondrial DNA in a patient with a novel deletion/duplication and high mutant load. J Med Genet. 2003;40:e15

18. von Kleist-Retzow JC, Cormier-Daire V, de Lonlay $\mathrm{P}$, et al. A high rate (20\%-30\%) of parental consanguinity in cytochrome-oxidase deficiency. Am J Hum Genet. 1998;63:428-435

19. Kirby DM, Crawford M, Cleary MA, Dahl H-HM, Dennett X, Thornburn DR. Respiratory chain complex I deficiency. An underdiagnosed energy generation disorder. Neurology. 1999;52:1255-1264

20. Rubio-Gozalbo ME, Dijkman KP, van den Heuvel LP, Sengers RCA, Wendel U, Smeitink JAM. Clinical differences in patients with mitochondriocytopathies due to nuclear versus mitochondrial DNA mutations. Hum Mutat. 2000;15:522-532

21. Ogasahara S, Engel AG, Frens D, Mack D. Muscle coenzyme Q deficiency in familial mitochondrial encephalomyopathy. Proc Natl Acad Sci U S A. 1989;86:2379-2382

22. Smeitink JAM, Loefflen JLCM, Triepels RH, Smeets RJP, Trijbels JMF, van den Heuvel LP. Nuclear genes of human complex I of the mitochondrial electron transport chain: state of the art. Hum Mol Genet. 1998;7:1573-1579

23. Wong L-JC, Liang M-H, Kwon H, Park J, Bai R, Tan D. Comprehensive scanning of the whole mitochondrial genome for mutations. Clin Chem. 2002;48:1901-1912
24. Robinson BH. Lacticacidemia. Biochim Biophys Acta. 1993;1182:231-244

25. Munnich A, Rötig A, Chretien D, et al. Clinical presentation of mitochondrial disorders in childhood. J Inherit Metab Dis. 1996;19:521-527

26. Munnich A, Rötig A, Chretien D, Saudubray J-M, Cormier V, Rustin P. Clinical presentations and laboratory investigations in respiratory chain deficiency. Eur J Pediatr. 1996;155:262-274

27. DiMauro S, Tay S, Mancuso M. Mitochondrial encephalomyopathies: diagnostic approach. Ann N Y Acad Sci. 2004;1011:217-231

28. Bernsen PLJA, Gabreels FJM, Ruitenbeek W, Sengers RCA, Stadhouders AM, Renier WO. Successful treatment of pure myopathy, associated with complex I deficiency, with riboflavin and carnitine. Arch Neurol. 1991;48:334-338

29. Vogel H. Mitochondrial myopathies and the role of the pathologist in the molecular era. J Neuropathol Exp Neurol. 2001;60:217-227

30. Sciacco M, Bonilla E. Cytochemistry and immunocytochemistry of mitochondria in tissue sections. Methods Enzymol. 1996;264:509-521

31. Shoffner JM. Maternal inheritance and the evaluation of oxidative phosphorylation diseases. Lancet. 1996;348:1283-1288

32. Smeitink J, van den Heuvel L. Human mitochondrial complex I in health and disease. Am J Hum Genet. 1999;64:1505-1510

33. Anan R, Nakagawa M, Miyata M, et al. Cardiac involvement in mitochondrial diseases. A study on 17 patients with documented mitochondrial DNA defects. Circulation. 1995;91:955-961

34. Towbin JA, Lipshultz SE. Genetics of neonatal cardiomyopathy. Curr Opin Cardiol. 1999;14:250-262

35. Holmgren D, Wåhlander H, Eriksson BO, Oldfors A, Holme E, Tulinius M. Cardiomyopathy in children with mitochondrial disease. Clinical course and cardiological findings. Eur Heart J. 2003;24:280-288

36. Casazza F, Morpurgo M. Progression of hypertrophic into a dilated left ventricle in Friedreich's ataxia. G Ital Cardiol. 1998;18:615-618

37. Momiyama Y, Atsumi Y, Ohsuzu F, et al. Rapid progression of cardiomyopathy in mitochondrial diabetes. Jpn Circ J. 1999;63:130-132

38. Pignatelli RH, McMahon CJ, Dreyer WJ, et al. Clinical characterization of left ventricular noncompaction in children. A relatively common form of cardiomyopathy. Circulation. 2003;108:2672-2678

39. Bleyl SB, Mumford BR, Thompson V, et al. Neonatal, lethal noncompaction of the left ventricular myocardium is allelic with Barth syndrome. Am J Hum Genet. 1997;61:868-872

40. Kelley RI, Cheatham JP, Clark BJ, et al. X-linked cardiomyopathy with neutropenia, growth retardation, and 3-methylglutaconic aciduria. J Pediatr. 1991;119:738-747

41. Bione S, D'Adamo P, Maestrini E, Gedeon AK, Bolhuis PA, Toniolo D. A novel X-linked gene, G4.5 is responsible for Barth syndrome. Nat Genet. 1996;12:385-389

42. Kartsounis LD, Troung DD, Morgan-Hughes JA, Harding AE. The neuropsychological features of mitochondrial myopathies and encephalomyopathies. Arch Neurol. 1992;49:158-160

43. Nissenkorn A, Zeharia A, Lev D, et al. Neurologic presentations of mitochondrial disorders. J Child Neurol. 2000;15:44-48

44. Turconi AC, Benti R, Castelli E, et al. Focal cognitive impairment in mitochondrial encephalomyopathies: a neuropsychological and neuroimaging study. J Neurol Sci. 1999;170:57-63

45. Uusima J, Remes AM, Rantala H, et al. Childhood encephalopathies and myopathies: a prospective study in a defined population to assess the frequency of mitochondrial disorders. Pediatrics. 2000;105:598-603

46. Jun AS, Brown MD, Wallace DC. A mitochondrial DNA mutation at nucleotide pair 14459 of the NADH dehydrogenase subunit 6 gene associated with maternally inherited Leber hereditary optic neuropathy and dystonia. Proc Natl Acad Sci U S A. 1994;91:6206-6210

47. Benecke R, Strumper P, Weiss H. Electron transfer complex I defect in idiopathic dystonia. Ann Neurol. 1992;32:683-686

48. Gold M, Rapin I. Non-mendelian mitochondrial inheritance as a cause of progressive genetic sensorineural hearing loss. Int J Pediatr Otorhinolaryngol. 1994;30:91-104

49. Edmonds JL, Kirse DJ, Kearns D, Deutsch R, Spruijt L, Naviaux RK. The otolaryngological manifestations of mitochondrial disease and the risk of neurodegeneration with infection. Arch Otolaryngol Head Neck Surg. 2002;128:355-362

50. Lincke CR, van den Bogert C, Nitjmans LG, Wanders RJ, Tamminga P, Barth PG. Cerebellar hypoplasia in respiratory chain dysfunction. Neuropediatrics. 1996;27:216-218 


\section{Clinical Spectrum, Morbidity, and Mortality in 113 Pediatric Patients With Mitochondrial Disease}

Fernando Scaglia, Jeffrey A. Towbin, William J. Craigen, John W. Belmont, E. O'Brian Smith, Stephen R. Neish, Stephanie M. Ware, Jill V. Hunter, Susan D. Fernbach, Georgirene D. Vladutiu, Lee-Jun C. Wong and Hannes Vogel Pediatrics 2004;114;925-931

DOI: $10.1542 /$ peds.2004-0718

\section{This information is current as of June 7, 2005}

$\begin{array}{ll}\begin{array}{l}\text { Updated Information } \\ \text { \& Services }\end{array} & \begin{array}{l}\text { including high-resolution figures, can be found at: } \\ \text { http://www.pediatrics.org/cgi/content/full/114/4/925 }\end{array} \\ \text { References } & \text { This article cites } 45 \text { articles, } 19 \text { of which you can access for } \\ \text { at: } & \text { http://www.pediatrics.org/cgi/content/full/114/4/925\#BIB } \\ \text { Subspecialty Collections } & \begin{array}{l}\text { This article, along with others on similar topics, appears in } \\ \text { following collection(s): } \\ \text { Infectious Disease \& Immunity } \\ \text { http://www.pediatrics.org/cgi/collection/infectious_diseas }\end{array} \\ & \begin{array}{l}\text { Information about reproducing this article in parts (figures, } \\ \text { tables) or in its entirety can be found online at: }\end{array} \\ \text { Permissions \& Licensing } & \text { http:/www.pediatrics.org/misc/Permissions.shtml } \\ & \begin{array}{l}\text { Information about ordering reprints can be found online: } \\ \text { http://www.pediatrics.org/misc/reprints.shtml }\end{array}\end{array}$

